\title{
ARTÍ́CULO
}

\section{PRESENCIALIDAD ONLINE COMO ELEMENTO DE ANÁLISIS EN LA RELACIÓN ESCUELA/COMUNIDAD. EL CASO DE LAS UNIDADES EDUCATIVAS DEL MILENIO EN TWITTER ${ }^{1}$}

\author{
GABRIEL CEVALLOS MARTÍNEZ ${ }^{*}$ \\ ORCID: https://orcid.org/0000-0002-6584-615X \\ VERÓNICA SOFÍA FICOSECO ${ }^{* *}$ \\ ORCID: https://orcid.org/0000-0003-1397-1968 \\ EDVALDO SOUZA COUTO ${ }^{* * *}$ \\ ORCID: https://orcid.org/0000-0002-2648-9399
}

RESUMEN: La presencialidad, originalmente tangible y uni-localizada, tiene ahora un carácter asíncrono y multisituado. Personas y colectivos crean presencialidades compuestas, habitando los espacios sociales online y offline en una continuidad sin suturas. La consideración de la espacialidad como dimensión integrada es una posición epistemológica largamente legitimada. El objetivo del artículo es explorar cómo las Unidades Educativas de Milenio - UEM construyen su presencia en Twitter para vincularse con la comunidad. Utilizamos el método cualitativo, descriptivo y analítico con base en análisis de redes sociales para analizar las cuentas referidas directamente a estas instituciones y, posteriormente, se describieron los mapas de red generados por las UEM como objeto de diálogo. Los resultados muestran que solamente 11 de 97 UEM poseen cuentas en Twitter, de aquellas, apenas dos tienen una actividad frecuente. Por otro lado, las UEM generan espacios de discusión atomizados, las menciones son coyunturales y sometidas principalmente a informes de entidades estatales.

Palabras clave: Presencialidad. Twitter. Unidades educativas. Milenio.

\footnotetext{
'Universidad Federal de Bahía, Salvador, Bahia, Brasil.

“Dr. Gabriel Cevallos Martínez (Doctor por la Universidad Federal de Bahía/UFBA).

E-mail:<gfcm83@hotmail.com>.

“" Dra. Verónica Sofía Ficoseco (Profesora Universidad Federal de Bahía/UFBA).

E-mail:<vsficoseco@gmail.com>.

"** Dr. Edvaldo Souza Couto (Profesor titular Universidad Federal de Bahía/UFBA).

E-mail:<edvaldosouzacouto@gmail.com>.
} 


\section{ONLINE PRESENCE AS AN ELEMENT OF ANALYSIS OF THE SCHOOL/COMUNITY RELATION. THE UNIDADES EDUCATIVAS DEL MILENIO CASE ON TWITTER}

ABSTRACT: Presentiality, originally tangible and uni-located, now has an asynchronous and multi-situated character. People and groups create complex presentialities, inhabiting online and offline social spaces in a continuum without sutures. The consideration of spatiality as an integrated dimension is a long-standing epistemological position. The objective of this article is to explore how the Unidades Educativas del Milenio - UEM build their Twitter presentiality to link with the community. We used qualitative, descriptive and analytical method based on analysis of social networks to analyze the Twitter accounts directly referred to these institutions and, later, the network maps generated by the UEM were described as an object of dialogue. Results show that only 11 out of 97 UEM have Twitter accounts, of those, only two have a frequent activity. Additionally, the UEM generate atomized spaces of discussion, and its mentions are conjunctural and mainly referred to reports from state entities.

Keywords: Presence. Twitter. Educational units. Millennium.

\section{INTRODUCCIÓN}

La presencialidad se ha desvinculado parcialmente de lo espacial y lo temporal, el "estar", gracias a la entrada de las Tecnologías de la Información y la Comunicación - TIC, tiene ahora un componente referido a la conexión a las redes, a las cuales se accede a través de diversas plataformas con enfoques, intencionalidades y limitaciones propios.

De manera constante, los sujetos van mutando su presencialidad tradicional a una presencialidad compuesta por lo encendido y lo apagado, lo offline y lo online, conviviendo en diversas plataformas. Esa presencia multisituada ocurre también para las organizaciones sociales, cuya existencia en plataformas de redes sociales es hoy en día generalizada, independiente de la naturaleza institucional y sus objetivos.

Desde esa perspectiva, el objetivo del artículo es analizar la presencialidad en Twitter de las Unidades Educativas del Milenio - UEM del Ecuador, instituciones educativas de enseñanza fundamental que el Gobierno ecuatoriano planteó desde el 2008 como modelo de escuela a nivel de talento docente, infraestructura física y equipamiento, para la superación del déficit educativo del país, contemplando la integración de las TIC en el proceso educativo. 
Las UEM encarnan, en teoría, la escuela que asume íntegramente las TIC en sus prácticas cotidianas tanto pedagógicas como organizacionales, cumpliendo el rol de dinamizadoras en la inclusión tecnológica de las comunidades en que se insertan, con la posibilidad de intercambiar experiencias a otras escuelas de su sector o zona de influencia, principalmente los territorios definidos como zonas de planificación en el Ecuador.

El análisis de la presencialidad de una institución o de cualquier sujeto social, tiene necesariamente carácter multisituado. En este trabajo, nos centraremos en analizar las interacciones de las UEM en la red Twitter, plataforma seleccionada por los siguientes motivos: primero, porque es la plataforma más utilizada por instituciones educativas (KIMMONS; VELETSIANOS; WOODWARD, 2017, p.98); segundo, porque se trata de la segunda red más utilizada en el Ecuador (MINISTERIO DE TELECOMUNICACIONES DEL ECUADOR, 2018); y tercero, porque permite conectividad con aplicativos de análisis de redes, metodología aplicada en este estudio. Las UEM son un campo de actuación educacional y de investigación relativamente nuevos, existiendo pocas investigaciones acerca del uso institucional de las redes sociales, por lo que los hallazgos obtenidos de este estudio se comparan principalmente con los resultados de una investigación enfocada a la plataforma Facebook.

Este artículo describe como estas instituciones se constituyen en sujeto y objeto de discusión en Twitter, trascendiendo los aspectos físicos institucionales tradicionales, determinándose algunos aspectos poco explorados de las relaciones entre escuela y comunidad. Tales aspectos pueden resultar fundamentales a la hora de comprender y canalizar nuevas demandas y de proponer nuevos roles y frentes de intervención.

Para analizar las UEM como sujetos en Twitter, se verificó la existencia de cuentas vinculadas a esas instituciones educativas y su grado de actividad, con el apoyo de una herramienta llamada Audiense. ${ }^{2}$ Para las UEM como objeto se capturaron por una semana con el aplicativo Gephi, ${ }^{3}$ menciones a las Unidades Educativas del Milenio. De esas menciones se consideraron usuarios relacionados, tweets, retweets y enlaces, con los que se crearon mapas de red, analizados en su configuración y métricas. Los resultados arrojaron poca presencia de las UEM como sujetos en Twitter, por otro lado, están presentes de manera persistente como tema informativo, especialmente por entidades públicas.

\section{LA PRESENCIALIDAD COMO DIMENSIÓN RELACIONAL MULTISITUADA}

El avance de las tecnologías digitales, ha posibilitado que surjan nuevas prácticas sociales y otras se vigoricen adquiriendo 
nuevas características. La vinculación actual de doble lado e infinitas formas entre las tecnologías digitales y la sociedad es uno de los objetos de estudio de la cibercultura, área de estudio que pretende analizar las dinámicas sociales, la generación de conocimiento y los efectos de la interacción e interactividad de las personas entre sí, y a través y con los maquinismos (LÉVY, 2014).

En ese nuevo escenario, el valor de las cosas no se vincula tanto a la producción de bienes y servicios sino a que información se obtiene y como esta puede utilizarse de manera estratégica. En el mundo globalizado de economía de la información, la red se convierte en modelo principal de organización social, capaz de promocionar comportamientos más integradores y democráticos, así como incrementar la vigilancia y el control de las personas.

En realidad, las nuevas formas de adquirir información y conocimiento establecen otras prácticas y formas de acción en lo cotidiano. Castells (2012) abordó una faceta de esas dinámicas a través del estudio de varios movimientos sociales cuya autoorganización fue posibilitada por las TIC, como la Primavera árabe o los indignados de España.

El autor, a través de sus observaciones, sostiene que "las redes de comunicación son un elemento indispensable en la práctica y organización de estos movimientos" (CASTELLS, 2012, p. 208), que se autoconvocaron a través de diversas redes sociales. Las redes como elemento de organización no se limitan a cuestiones coyunturales, sino que tienen ese mismo papel en el cotidiano de personas, colectivos e instituciones que son se organizan y comunican en Internet.

Por supuesto, las instituciones son colectivos de personas con propósitos definidos, que forman parte y se expresan en el escenario de red. La identidad es una construcción colectiva que, como todo lo demás y según lo preveía Negroponte (1995, p. 64), pasa por “puntos específicos del espacio y del tiempo en donde los bits se transformarán en átomos y viceversa".

Así, cada pedazo del "ser" toma forma de textos, imágenes, sonidos y videos, trozos que por diversos medios son transformados, mixeados, compartidos, gustados y juzgados, es así, como la identidad y la presencia de las organizaciones se reconfiguran en la red, en fluidez dialógica, estando presente tanto en la dimensión tradicional off-line como en la nueva dimensión posibilitada por las tecnologías digitales on-line. (HINE, 2015).

La red misma, expresada en los diversos maquinismos y en el software que los controla, es un actor que interviene directamente en nuestra forma de comunicarnos, de ahí la importancia de analizar, no 
solamente la acción de personas en la red, sino como la red implica a personas e instituciones, a través de sus diversas plataformas. De ahí la importancia de registrar, no apenas como las personas actúan entre sí, sino como las plataformas median y son actuantes en la red, definiendo espacios y sitios con particularidades propias. Aquella presencialidad en red que diferencia sus espacios a través de páginas y plataformas, posee fronteras difusas y permeables que permiten el diálogo entre las aplicaciones, volviendo multidimensional la presencia, ya que una misma expresión muta es transformada por equipos telemáticos para atravesar, al mismo tiempo, diversos sitios. Esto contrasta con la presencialidad tradicional que, si bien ocurre también en diversos espacios, estos son poco permeables y monosituados. (LEMOS, 2013)

Los mensajes, en sí mismos, tienen otros énfasis en la red, adquiriendo carácter universal y de libre compartición, atemporalidad, recuperabilidad y mutabilidad al mezclarse con otros objetos. De esta manera, la presencia en red se vincula también con la posibilidad de transformar aquello que se es y se hace, para vincularlo con los demás, de esta forma la presencia en la red expresa su visibilidad.

Sumados al número telefónico, la dirección física y el edificio, los colectivos cuentan ahora con correo electrónico, página web, aplicaciones, servicios, perfiles y canales de comunicación, con los cuales interactúan con la comunidad. Las nuevas formas de mantenerse en contacto con su contexto conforman sistemas de información estratégicos (COHEN; ASÍN, 2000) que de manera dialógica permiten ubicar las tendencias, necesidades, hábitos y opiniones del sector al que se quiere atender.

De esta forma, el flujo de información es la variable principal de la presencia, las posibilidades de crear y compartir información para generar diálogo, ubican a las instituciones de manera preferencial en la conciencia colectiva, haciendo fluida también la relación con la comunidad, situación deseable para aquellas que se dedican al quehacer educativo.

\section{UNIDADES EDUCATIVAS DEL MILENIO}

Las UEM conforman una de las iniciativas que impulsa el Gobierno ecuatoriano, a través de su Ministerio de Educación para elevar la cobertura educativa del país. Forman parte del proyecto "Nueva infraestructura escolar", cuyos antecedentes se remontan al año 2005, cuando el Ecuador suscribió la Declaración del Milenio, en la Organización de las Naciones Unidas (ONU). 
Los Objetivos del Milenio (ODM), actualizados posteriormente como Objetivos de Desarrollo (ODS), con horizonte de cumplimiento al 2030, establecen que todos los países signatarios se comprometerán a impulsar la educación primaria universal, reconocida como derecho para todos los niños y niñas.

La iniciativa UEM se fundamenta en cuatro características principales: Calidad, Tecnología, Integración con la comunidad e Infraestructura. La Calidad es entendida como la participación de todos los actores en los planes educativos, la promoción de la evaluación continua, enfocada a la mejora y la creación de proyectos de diversos niveles y propósitos. Las Tecnologías de la información y comunicación integradas efectivamente para potenciar los procesos de enseñanza - aprendizaje (UNESCO, 2013) y la participación, diálogo y corresponsabilidad de los actores externos de la comunidad educativa. Integración de los padres de familia y actores de la comunidad para planear, ejecutar y evaluar las acciones dentro de la institución, y trabajar en el aporte de la escuela a la vida comunitaria, e Infraestructura caracterizada por criterios etnográficos de la zona de influencia, así como por accesos y pasos necesarios para la inclusión de personas con discapacidades.

Según los datos del Ministerio de Educación del Ecuador, actualizados al 1 de febrero de 2018, 97 UEM se encuentran en funcionamiento, y 38 están en proceso de construcción. Los criterios de ubicación de estas instituciones priorizan poblaciones de altos niveles de pobreza, baja oferta educativa y resultados académicos bajos en las pruebas de desempeño de educación media llamadas "Ser Bachiller" (MINISTERIO DE EDUCACIÓN DEL ECUADOR, 2010). De esta forma se eleva el impacto de la intervención educativa en la población beneficiada.

Siendo uno de los ejes de las UEM la participación de la comunidad, se analizó cómo el potencial de las redes sociales es efectivizado por estas instituciones. Se seleccionó Twitter en vista de su énfasis en el estilo de comunicaciones breves, fluidas e instantáneas, lo cual pone de manifiesto su potencial para romper la unidireccionalidad de los vínculos entre institución y comunidad, y al mismo tiempo haría posible establecer modelos de interacción dialógica, más horizontales en comparación con las formas tradicionales de comunicación institucional (KIMMONS; VELETSIANOS; WOODWARD, 2017, p.108). Esta relación es particularmente valiosa para que las UEM consigan transformarse en centros de desarrollo para las comunidades en el contexto en que están insertas. 


\section{METODOLOGÍA}

Para el presente estudio se utilizó el método cualitativo descriptivo y analítico (MINAYO, 2011), a través de un análisis empírico en Twitter aplicado a las UEM, en dos dimensiones analíticas complementarias: las UEM como sujetos y como objeto de discusión en la plataforma.

Para evaluar la presencia de la UEM como sujetos, se vinculó el "Ser" con la existencia de cuentas de las instituciones en Twitter y con el uso de las UEM para describirse o desarrollar identidad. En otras palabras, se definió la presencialidad de las UEM como sujeto, al conjunto de cuentas cuyo nombre (name), nombre de usuario (username) o descripción de cuenta (Bio), hacen referencia a las unidades educativas del milenio. Para determinar las condiciones de búsqueda se realizó previamente una exploración en extenso, para observar como las cuentas de las instituciones educativas son definidas.

Por ejemplo, al utilizar las palabras "Unidad", "Educativa" y "Milenio", no se obtuvo ningún resultado, no existiendo coincidencias ni con el nombre de usuario, nombre de la cuenta, ni con la descripción de ninguna cuenta registrada hasta la fecha. Por ese motivo, se optó por dejar solamente la palabra "Unidad" para ampliar el número de resultados.

En la nueva búsqueda, pudo observarse que en el campo "Bio" y "Nombre" la palabra "Unidad" es usada constantemente, dejando las siglas "UE", o las palabras unidas "unidadeducativa", para el nombre de usuario de la unidad educativa.

Adicionalmente, se observó que varios de los resultados obtenidos no se vinculaban a cuestiones educativas y estaban más ligados a colectivos de acción política, por ejemplo, la cuenta de "UnidadRevolucionaria" (@35PAIS_UR), por ese motivo, se eligió condicionar las palabras de búsqueda a "Unidad educativa".

Definido lo anterior, la búsqueda en Audiense fue ejecutada en marzo de 2018 y la configuración consideraría todas las cuentas que cumplan con las siguientes condiciones:

- La Bio contiene la frase "Unidad educativa" y el nombre contiene "Unidad educativa" o "UE"

- La Bio contiene la sigla "UEM" y el nombre contiene "Unidad educativa" o "UE"

- La Bio contiene "U.E" o "U.E." y el nombre contiene "Unidad educativa" o "UE"

- El nombre de usuario contiene "Unidadeducativa" y es una cuenta creada en Ecuador

- La Bio contiene "Unidad", "Educativa" y "Milenio" 
Si bien la búsqueda pudo configurarse desde un primer momento a cuentas en Ecuador para delimitar la muestra, la exploración previa en Twitter permitió observar que varias de las cuentas creadas alrededor de las Unidades Educativas no tienen declarado su lugar de origen, en ese sentido, se prefirió ampliar los filtros para perder la menor cantidad posible de información.

Por otrolado, la exploración fue también de utilidad para verificar que una de las cuentas que aparecían en Twitter, la correspondiente a la Unidad Educativa del Milenio "Jatun Kuraka" (@JatunUnidad), no aparecía en las cuentas halladas por Audiense, debido a que esta cuenta no tenía seguidores. Por lo anterior, y siempre intentando ser inclusivos en la muestra inicial, las 418 cuentas encontradas por Audiense, se sumaron a 25 cuentas importadas desde la búsqueda de Twitter, alcanzando un grupo base de 443 cuentas, cuyo Bio, Nombre o Nombre de usuario se refería de alguna manera a una Unidad educativa.

Posteriormente, se realizó una depuración de las cuentas encontradas, retirando inicialmente todas las cuentas que tienen declaradas localización diferente a Ecuador, y, separando después aquellas cuentas referidas a Instituciones educativas diferentes de las UEM. De la primera depuración fueron separadas 74 cuentas de Venezuela, 22 de España, seis de Brasil y una de Colombia, quedando 340 cuentas. En una segunda depuración se verificó la Bio y la localización por ciudades de cada cuenta. Cuentas de ciudades de Venezuela, Colombia, Perú o Brasil, o a la Universidad Europea de Madrid (UEM), por ejemplo, fueron retiradas; al finalizar esta segunda depuración quedaron 163 cuentas.

La tercera depuración, se enfocó en seleccionar las cuentas que se relacionaban de forma directa con las Unidades Educativas del Milenio, para el efecto se revisó una a una los datos de las cuentas existentes, siendo las principales dificultades en el análisis la información parcial de las cuentas y la diversidad en el modo de referirse a la vinculación con la Unidad Educativa del Milenio.

En cuanto a la información parcial, en la mayoría de los casos no se ubicaba la naturaleza de la institución referida, pocas veces se colocaba si la institución era de tipo particular, fiscal o fiscomisional, peor aún si se trataba de una Unidad Educativa del Milenio. De igual forma, sin la localización dentro del Ecuador, existió dificultad para distinguir la institución mencionada, especialmente en instituciones homónimas.

Por otro lado, al referirse a las unidades educativas en general los usuarios utilizaban siglas de diversos formatos (UE, U.E, U.E.), se utilizaban términos como "escuela" o "colegio", o solamente la 
palabra "unidad". De esta forma, se retiraron las cuentas personales que en su descripción o Bios hacían referencia a instituciones educativas que no eran las UEM, saliendo cuentas que se referían a 27 instituciones particulares, 2 fiscomisionales y 36 fiscales.

Por otro lado, se separaron también las cuentas oficiales de 43 instituciones particulares, dos fiscomisionales, 14 fiscales y dos municipales, además de una cuenta referida a una universidad pública. Adicionalmente, se encontraron tres cuentas de colectivos vinculados a unidades educativas, una del Club de periodismo, otra de una liga anti-drogas y la cuenta del Gobierno estudiantil de una entidad.

Terminada esta tercera depuración, quedaron 34 cuentas de Twitter, algunas de ellas son cuentas oficiales de las UEM, mientras que otras se refieren a las unidades al describirse. Como se observa, se amplió la búsqueda al máximo intentando cubrir cualquier posibilidad de coincidencia con la búsqueda de las UEM como sujetos en Twitter. Sin embargo, la diversidad de las menciones al expresarse en la plataforma y la ambigüedad del lenguaje, se admiten como limitantes de lo hallado.

De las 34 cuentas se observó si estaban verificadas, ${ }^{4}$ y se las caracterizó según nombre y descripción, observando cuales de estas cuentas eran personales que mencionaban a las UEM, o aquellas que eran institucionales, ya sean o no oficiales. Posteriormente se obtuvieron métricas de las cuentas personales e institucionales, por separado, caracterizando estos grupos según el tipo de actividad y frecuencia de posteo, los principales datos de cada cuenta se exportaron a hoja de datos a través de un plug-in de Chrome llamado Twlets. ${ }^{5}$

Para poder comparar por separado la actividad de las cuentas encontradas, se dividió el número total de Tweets de cada cuenta por su tiempo de existencia en años, de esta forma, al compartir la misma base, se podían cotejar los datos de cuenta a cuenta. También se caracterizaron las imágenes utilizadas para representar cada perfil.

Para analizar a las UEM, como objeto de discusión en Twitter, se decidió capturar, de forma exploratoria, todos los tweets que hacían referencia a las palabras "Unidad", "Educativa" y "Milenio" entre las 9h30 del 7 de marzo, hasta las 17 h00 del 13 de marzo de 2018. Ese periodo fue seleccionado porque en ese mes se celebran en todas las instituciones de la sierra ecuatoriana las "Casas Abiertas del Programa de Participación Estudiantil” por lo cual hay mayor posibilidad de comunicación entre la comunidad y la UEM. Para la captura de Tweets se utilizó la aplicación Gephi, y el plugin "Twitter streaming importer".

Gephi es una aplicación para la implementación y desarrollo de Mapas de red, utilizada especialmente en análisis de redes sociales. A 
través de diversas configuraciones, se adecua los llamados mapas de red, gráficos o esquemas que representan la actividad de las personas y sus mensajes (RECUERO; BASTOS; ZAGO, 2015). En este caso, el plugin captura los tweets, según lo configurado, pudiéndose hacer seguimiento a la actividad a través de palabras clave, seguimiento de usuarios, territorio global e incluso con emoticones. La captura se configuró en modo: "Full Smart network", es decir se capturan no solamente los mensajes, sino los usuarios, menciones, retweets, enlaces y hashtags vinculados. De esta forma se tiene una mayor cantidad de datos que permitan interpretar no solamente como las UEM son abordadas, sino por quién y en que formas. ${ }^{6}$

Los datos obtenidos configuraron una muestra de intercambios alrededor de las UEM, representada como un mapa de red, involucrando a los autores del mensaje, la naturaleza de estos y la forma en que se conectan. Al final se obtiene un gráfico, cuyos actores son llamados nodos, y sus acciones los vinculan y son representadas a través de líneas, llamadas aristas.

Por ejemplo, la representación de un mensaje o Tweet que " $\mathrm{A}$ " envía colocando un hashtag y un enlace o imagen, involucra a cuatro nodos: el nodo del que escribe, el mensaje en sí mismo (T), el hashtag (\#) y la multimedia incluida (M). Estos nodos se enlazan entre sí a través de tres aristas, como muestra la figura 1. Nótese que la flecha indica el direccionamiento del vínculo, es decir muestra el nodo que origina la acción y aquel que la recibe, lo cual es propio de las redes llamadas dirigidas o direccionadas.

En el análisis se aplicaron las siguientes métricas de red: grado, diámetro, densidad y modularidad. El grado es el número de otros nodos a los que un actor dado se encuentra vinculado directamente. En redes direccionadas, hay dos métricas vinculadas al grado, la llamada "Soporte" o prestigio de entrada - in-degree, y la llamada "Influencia" o prestigio de salida - out-degree. (HANNEMAN, 2014; LARROSA, 2016) La red de la figura 1, "T", por ejemplo, posee un grado de Influencia de 2 y de Soporte de 1 . El grado medio, en redes direccionadas, corresponde a la sumatoria de los grados de Soporte e Influencia, de todos los nodos, dividido para dos veces el número de nodos. En cuanto a la red estudiada, el grado medio tiene un valor de 1,388; es decir, los nodos de la red usan mayormente uno o dos enlaces para vincularse entre sí, evidenciándose que la red muestra pocas alternativas de comunicación directa entre nodos.

Sobre la extensión de la red, la medida elemental se conoce como diámetro, que determina la distancia existente entre los nodos 
más alejados de la red, tomándose cada arista como medida 1 de distancia. Nuestra red muestra un diámetro de cinco, separándose los nodos más distantes por cinco pasos, lo que revela una red compacta, o de poca extensión.

La plena conexión de red es aquella donde se han alcanzado todas las posibles conexiones entre sus nodos, a mayor cantidad de conexiones posibles cubiertas, más se acerca una red a la plena conexión y adquiere mayor densidad. Una red densa es aquella cuyos nodos están fuertemente ligados pues poseen varios caminos para vincularse, la densidad se mide como la relación entre las conexiones existentes y el total de conexiones posibles.

FIGURA 1. Ejemplo de Mapa de red para Twitter

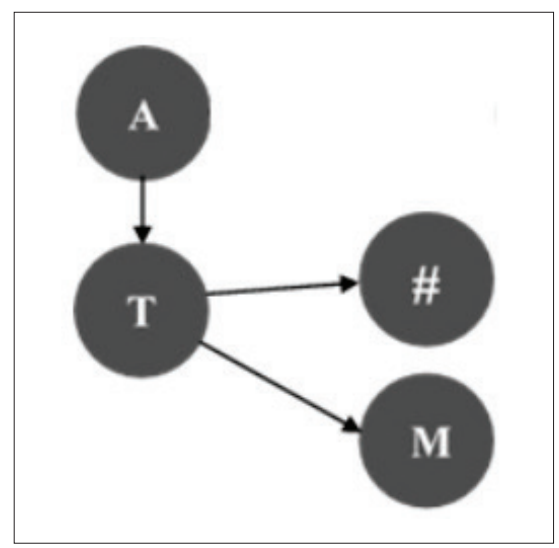

Elaborado por los autores.

La información de las métricas de red se utilizó para establecer los niveles y tipo de repercusión de las UEM dentro de la plataforma. Además, otro tipo de información se pudo extraer de la red, la llamada modularidad o existencia de sub-comunidades o clusters. En todo tipo de redes es común que existan vinculaciones más fuertes entre ciertos nodos, lo cual evidencia comunidades más pequeñas dentro de la red mayor. Por ejemplo, dentro de una red de apoyo político es probable que agrupaciones locales, al tiempo que participan del diálogo más amplio, mantengan vinculaciones más fuertes, como mayor cantidad de menciones, retweets y uso coordinado de hashtags que fortalecen su presencialidad en la red.

La identificación de modularidad se realizó a través de algoritmos matemáticos que, de manera sencilla, comparan las vinculaciones de la red con patrones aleatorios de nodos y vínculos. Si el algoritmo identifica que lo encontrado supera significativamente 
a lo aleatorio, para un conjunto de nodos dado, detecta la existencia de una sub-comunidad. Según investigaciones en el área (PÉREZ BELTRÁN; VALERIO UREÑA; RODRÍGUEZ-ACEVES, 2015), un valor de modularidad superior a 0,4 indica la existencia de clusters en la red. El análisis de modularidad permitió describir y analizar cómo las UEM son discutidas, y que tipo de relaciones generan dentro de la plataforma Twitter.

Como opción metodológica se mantuvieron los datos originales de las cuentas analizadas, debido a que la muestra obtenida se compone em su otalidade de cuentas abiertas, de acceso público em Twitter.

\section{RESULTADOS Y ANÁLISIS}

Con relación a la primera dimensión analítica, se estableció que ninguna de las cuentas es verificada, lo que indica que las cuentas vinculadas a las UEM no poseen peso en la plataforma. Además, las cuentas que se refieren de forma directa a las UEM no necesariamente son oficiales. La tabla 1 muestra el username, el nombre y la descripción de las trece cuentas que se refieren directamente a las UEM, en cada una de las tablas del presente trabajo, se mantienen fielmente los caracteres y énfasis utilizados.

TABLA 1. Cuentas institucionales de UEM en Twitter

\begin{tabular}{|c|c|c|}
\hline Nombre de usuário & Nombre & Descripción \\
\hline JatunUnidad & $\begin{array}{l}\text { Unidad Educativa Jatun } \\
\text { Kuraka Otavalo }\end{array}$ & \\
\hline r28mayo & UEF.R28MAYO & $\begin{array}{l}\text { UNIDAD EDUCATIVA FISCAL RÉPLICA } \\
28 \text { DE MAYO }\end{array}$ \\
\hline Replicanid & Réplica Nicolás & $\begin{array}{l}\text { Unidad Educativa del Milenio Réplica } \\
\text { Nicolás Infante Díaz }\end{array}$ \\
\hline SigUnidad & Unidad Educativa Sig & \\
\hline SUMKYACHANAWASI & $\begin{array}{l}\text { UEM } \\
\text { SUMAKYACHANAWASI }\end{array}$ & UEM Sumak Yachana Wasi \\
\hline UEFR28DEMAYO & UEFR 28 DE MAYO & $\begin{array}{l}\text { UNIDAD EDUCATIVA FISCAL REPLICA } \\
28 \text { DE MAYO }\end{array}$ \\
\hline UEM_JJCZ & UEM JuanJ Castelló Z & $\begin{array}{l}\text { Unidad Educativa del Milenio, dedicados } \\
\text { a la formación de jóvenes emprendedores. }\end{array}$ \\
\hline
\end{tabular}




\begin{tabular}{|l|l|l|}
\hline Uem10N & UEM10N & $\begin{array}{l}\text { La Unidad Educativa del Milenio 10 de } \\
\text { Noviembre fue creada el } 20 \text { de mayo del } \\
\text { 2014. Esta ubicada en Zamora Chinchipe, } \\
\text { cantón Yantzaza - Los Encuentros }\end{array}$ \\
\hline UEMBernardoVald & $\begin{array}{l}\text { Unidad Educativa } \\
\text { del Milenio Bernardo } \\
\text { Valdivieso }\end{array}$ & $\begin{array}{l}\text { La Unidad Educativa del Milenio Bernardo } \\
\text { Valdivieso es una institución laica que } \\
\text { acoge a la niñez y juventud, para brindar } \\
\text { una Educación de Calidad }\end{array}$ \\
\hline UEMCAAA2 & UEMCAAA & $\begin{array}{l}\text { Unidad Educativa del Milenio Carlos } \\
\text { Alberto Aguirre Avilés. }\end{array}$ \\
\hline UemNelaMartínez & Nela Martínez & $\begin{array}{l}\text { Unidad Educativa del Milenio Nela } \\
\text { Martinez Espinosa empezó a funcionar en } \\
\text { el periodo lectivo 2011 - 2012 con una } \\
\text { visión de educación con calidad y calidez }\end{array}$ \\
\hline UERosaGmezC491 & U.E.RosaGómezC.491 & $\begin{array}{l}\text { Unidad Educativa Experimental del Milenio } \\
\text { Rosa Gómez de Castro 491, creada el 13 } \\
\text { de abril del 2007, en la parroquia Tarqui, } \\
\text { con su jornada Diurna e EGB }\end{array}$ \\
\hline wirc07 & Réplica Montúfar & $\begin{array}{l}\text { Unidad Educativa del Milenio inaugurada } \\
\text { en junio del 2014 }\end{array}$ \\
\hline
\end{tabular}

Elaborado por los autores.

De las 13 cuentas, dos no poseen descripción, el resto utiliza el espacio para colocar el nombre completo institucional, y en algunos casos, la fecha de inauguración. El proyecto de las UEM es relativamente reciente, habiendo sido la primera de ellas inaugurada en el año de 2008. Una de las cuentas corresponde a la Unidad Educativa Rosa Gómez de Castro, institución fiscal que no es parte de las UEM, como se coloca erróneamente. Por otro lado, la Unidad Educativa del Milenio "Réplica 28 de Mayo" posee dos cuentas, una de ellas inactiva hace casi 5 años (@UEF.R28MAYO).

En resumen, de las 97 Unidades Educativas del Milenio en funcionamiento, solamente once han creado o tienen cuentas institucionales en la plataforma. En cuanto a la representación visual nueve de las cuentas seleccionan como foto de perfil a los escudos institucionales, tres eligen grupos de estudiantes y docentes y una pone la imagen de su edificio.

$\mathrm{Al}$ analizar la visibilidad de las cuentas, los valores son bajos, ninguna de las cuentas supera los 50 seguidores, y nueve de ellas $(69 \%)$ siguen a menos de 50 personas. Solamente tres cuentas $(23 \%)$ han reportado actividad en el pasado mes, mientras que seis de ellas 
no han emitido un tweet desde hace más de un año. Adicionalmente solo tres cuentas tienen más seguidores que personas a las que siguen, lo cual muestra que la comunidad educativa no usa este canal para involucrarse con la institución, probablemente por falta de condiciones o campañas informativas. En cuanto a la existencia, la cuenta más nueva corresponde a la Unidad Educativa del Milenio Bernardo Valdivieso, que tiene 18 días desde su creación a la fecha del análisis, mientras que la cuenta de la UEM Juan José Castello tiene casi cinco años de creación. Sobre la actividad de las cuentas, la tabla 2 muestra el total de tweets, tiempo de vida y los tweets por año de cada cuenta a corte 15 de marzo de 2018.

TABLA 2. Tweets por año de cuentas de UEM en Twitter

\begin{tabular}{|l|l|l|l|}
\hline Nombre & $\begin{array}{l}\text { Existencia } \\
\text { (Años) }\end{array}$ & $\begin{array}{l}\text { Tweets } \\
\text { totales }\end{array}$ & $\begin{array}{l}\text { Tweets } \\
\text { por año }\end{array}$ \\
\hline Nela Martínez & 6,00 & 22 & 3,67 \\
\hline Réplica Montúfar & 1,64 & 835 & 507,77 \\
\hline Réplica Nicolás & 3,86 & 2 & 0,52 \\
\hline U.E.RosaGómezC.491 & 6,17 & 1 & 0,16 \\
\hline UEF.R28MAYO & 4,45 & 10 & 2,25 \\
\hline UEFR 28 DE MAYO & 3,82 & 164 & 42,91 \\
\hline UEM JuanJ Castelló Z & 4,74 & 10 & 2,11 \\
\hline UEM SUMAKYACHANAWASI & 0,74 & 23 & 31,25 \\
\hline UEM10N & 2,76 & 3 & 1,09 \\
\hline UEMCAAA & 1,27 & 0,31 & 563,24 \\
\hline Unidad Educativa del Milenio Bernardo Valdivieso & 0,05 & 0 & 160,00 \\
\hline Unidad Educativa Jatun Kuraka Otavalo & 3,19 & 28,93 \\
\hline Unidad Educativa Sig & 0,00 \\
\hline
\end{tabular}

Elaborado por los autores. 
Dos cuentas se separan totalmente del grupo, se trata de la cuenta de la UEM Carlos Alberto Aguirre Avilés con 563 Tweets por año y la UEM Réplica Juan Pío Montúfar con 508 Tweets por año, la primera con actividad en los últimos dos meses y la segunda dentro del último. Ambas cuentas tienen más de un año, y demuestran mantenerse en actividad, siendo también las que tienen más seguidores con 29 y 33 , respectivamente.

A esas dos cuentas les sigue la cuenta de la UEM Bernardo Valdivieso, con una proyección anual, en lugar de constatación, ya que tiene menos de un mes de existencia. En sus 18 días de existencia esta cuenta ha generado ocho tweets que proyectados a un año equivaldrían a 160. Sin embargo, esto no asegura que la actividad se mantenga a ese nivel, posterior seguimiento a esta y otras cuentas de creación reciente permitiría corroborar la proyección.

La cantidad de Tweets por año va decreciendo en el resto de cuentas, desde 43 de una de las cuentas de la UEM Réplica 28 de Mayo, hasta 0 de la cuenta UEM Técnico Sigchos. Adicionalmente, solo tres cuentas se refieren a enlaces o páginas web, una página de Facebook de la UEM Réplica 28 de mayo (@UEF.R28MAYO), la página del Ministerio de Educación en la cuenta de la UEM Réplica Nicolás Infante Díaz, y una página institucional completa de la UEM Bernardo Valdivieso (@UEMBernardoVald).7

En síntesis, el conjunto de cuentas vinculadas a las UEM de forma directa tiene baja presencialidad, con poca cantidad de seguidores. Solo dos cuentas muestran estar generando información de manera relativamente frecuente, y otra, de reciente creación, ha mostrado actividad importante. Esta situación junto con la página institucional evidenciaría acciones de esta institución en particular por visualizarse en la red, sin embargo, por la condición novel de estas iniciativas aún es necesaria mayor observación.

En cuanto a las cuentas personales, se encontraron 21 que citan a las UEM, en especial en la descripción, se trata principalmente de rectores y docentes que colocan su vinculación profesional con la institución en su cuenta. En total se tienen 13 docentes de diversas UEM, cinco estudiantes, un ex profesor, una ex rectora y un vicerrector.

La tabla 3 muestra el nombre de usuario, nombre y descripción de estas cuentas, manteniéndose los énfasis, mayúsculas y lenguaje de manera fiel a como han sido colocadas en las cuentas analizadas. 
TABLA 3. Cuentas vinculadas a las UEM en Twitter

\begin{tabular}{|c|c|c|}
\hline $\begin{array}{l}\text { Nombre de } \\
\text { usuario }\end{array}$ & Nombre & Descripción \\
\hline a_bzn2013 & Alfredo & $\begin{array}{l}\text { Docente titular de la Unidad Educativa del Milenio } \\
\text { Lcda. Olga Campoverde }\end{array}$ \\
\hline ArielJarrin & @ ariel & unidad educativa del milênio \\
\hline Boscoguiatour & Manuel Rivadeneira & $\begin{array}{l}\text { Profesor en la Unidad Educativa Dr. Camilo } \\
\text { Gallegos D. }\end{array}$ \\
\hline Buelewellington & Wellington Buele & $\begin{array}{l}\text { Licenciado en Ciencias de la Educación. Docente } \\
\text { de la Unidad Educativa del Milenio Lcda. Olga } \\
\text { Campoverde. Huaquillas Distrito de Educación } \\
\text { 07D05. }\end{array}$ \\
\hline carmenenriquet1 & Carmen Aguilar & $\begin{array}{l}\text { Ex Docente de la Unidad educativa del Milenio DR. } \\
\text { Alfredo Raúl Vera Vera }\end{array}$ \\
\hline Danny_Arevalo17 & Danny Arevalo Pilay & $\begin{array}{l}\text { SI.... YO, El mismo de siempre... } \\
\text { Prof. de Educación Física en la Unidad Educativa } \\
\text { del Milenio } 2 \text { de Agosto. en el Cantón Isidro Ayora. }\end{array}$ \\
\hline Eddycriss5 & Eddy Mora Castañeda & profesor de la unidad educativa Milenio \\
\hline fannyro41100440 & Fanny Rodríguez S. & $\begin{array}{l}\text { Rectora de la Unidad Educativa del Milenio Réplica } \\
24 \text { de Mayo }\end{array}$ \\
\hline Humbcordova & Humberto Cordova & $\begin{array}{l}\text { I am a English teacher at Unidad Educativa del } \\
\text { milenio Cerezal Bellavista. }\end{array}$ \\
\hline Hvchuquimarca & Homer Chuquimarca & $\begin{array}{l}\text { Vicerector de la Unidad Educativa del Milenio } 5 \\
\text { de Junio }\end{array}$ \\
\hline IlianaYagual & Iliana Yagual & $\begin{array}{l}\text { Estudiante de secundaria en Unidad Educativa del } \\
\text { Milenio Experimental Dr. Alfredo Raul Vera Vera. }\end{array}$ \\
\hline
\end{tabular}




\begin{tabular}{|c|c|c|}
\hline Jhonmathimale & Maria Elena & $\begin{array}{l}\text { Licenciada en Fisico Matematico... trabajo en } \\
\text { Unidad Educativa del Milenio Flavio Alfaro... naci } \\
\text { el } 30 \text { de abril de } 1984\end{array}$ \\
\hline JoaTugata & Joanna mishel & $\begin{array}{l}\text { vivo en huaquillas, estudio en la unidad educativa } \\
\text { del milenio lic. olga campoverde c. }\end{array}$ \\
\hline KaterineAnchund & Katerine Anchundia & $\begin{array}{l}\text { Docente en la Unidad Educativa del Milenio Lcda. } \\
\text { Olga Campoverde. }\end{array}$ \\
\hline levm83 & Luis Edgar Vinueza & Docente UEM Sumak Yachana Wasi \\
\hline Leytom & Leytom & Estudiante de la Unidad Educativa del Milenio \\
\hline Luisbar23143150 & Luis barba & $\begin{array}{l}\text { Estudio el unidad educativa del milenio Dr:Camilo } \\
\text { gallegos Domínguez }\end{array}$ \\
\hline Meibol23 & Mabelita & $\begin{array}{l}\text { Docente de la Unidad Educativa del Milenio Simón } \\
\text { Plata Torres }\end{array}$ \\
\hline narciso_1956 & narciso de jesus & $\begin{array}{l}\text { Lcdo. Narciso Saltos Palacios Docente Unidad } \\
\text { Educativa Milenio }\end{array}$ \\
\hline Patricgp & Patricio Garrochamba & $\begin{array}{l}\text { Docente de la Unidad Educativa del Milenio } \\
\text { Bernardo Valdivieso }\end{array}$ \\
\hline SilviaGaibor05 & Silvia Gaibor & $\begin{array}{l}\text { Docente de la Unidad Educativa del Milenio } \\
\text { Carmelina Granja Villanueva }\end{array}$ \\
\hline
\end{tabular}

Elaborado por los autores.

En estas cuentas, las UEM se utilizan para definir identidad, en pocos casos se acompañan datos adicionales, colocándose a veces la fecha de nacimiento o la materia impartida, en general los titulares de estas cuentas hacen de las UEM sujetos para autodefinirse. Al observar las métricas de visibilidad y actividad se evidencian índices bajos, solamente dos cuentas superan los 50 seguidores y apenas una los 100; así mismo, solo dos cuentas tienen mayor número de seguidores que personas a las que siguen. La tabla 4, muestra los tweets por año de estas cuentas. 
TABLA 4 Tweets por año de cuentas vinculadas a las UEM

\begin{tabular}{|l|l|l|l|}
\hline Nombre & $\begin{array}{l}\text { Tiempo de existencia } \\
\text { (Años) }\end{array}$ & $\begin{array}{l}\text { Tweets } \\
\text { totales }\end{array}$ & $\begin{array}{l}\text { Tweets } \\
\text { por año }\end{array}$ \\
\hline Alfredo & 4,65 & 3 & 0,65 \\
\hline @ Ariel & 3,34 & 29 & 8,68 \\
\hline Manuel Rivadeneira & 3,82 & 0 & 0,00 \\
\hline Wellington Buele & 5,48 & 176 & 32,11 \\
\hline Carmen Aguilar & 6,00 & 96 & 16,00 \\
\hline Danny Arevalo Pilay & 4,49 & 1064 & 236,88 \\
\hline Eddy Mora Castañeda & 3,66 & 3 & 0,82 \\
\hline Fanny Rodríguez S. & 4,62 & 76 & 16,44 \\
\hline Humberto Cordova & 5,89 & 0 & 0,00 \\
\hline Homer Chuquimarca & 1,99 & 1 & 0,50 \\
\hline lliana Yagual & 4,43 & 2 & 0,45 \\
\hline Maria Elena & 5,00 & 32 & 6,40 \\
\hline Joanna Mishel & 5,87 & 1 & 0,17 \\
\hline Katerine Anchundia & 6,50 & 29 & 4,46 \\
\hline Luis Edgar Vinueza & 4,83 & 13 & 2,69 \\
\hline Leytom & 6,51 & 0 & 0,00 \\
\hline Luis barba & 0,26 & 19 & 72,77 \\
\hline Mabelita & 7,99 & 21 & 2,63 \\
\hline narciso de jesus & 5,00 & 9 & 1,80 \\
\hline Patricio Garrochamba & 5,59 & 8,76 \\
\hline Silvia Gaibor & 1,64 & 615,48 \\
\hline
\end{tabular}

Elaborado por los autores.

En cuanto a la actividad, los resultados son más heterogéneos, mientras cinco de las cuentas han tweeteado en las últimas 24 horas, seis de ellas no han enviado un mensaje desde hace un año, quedando las demás entre esos dos estados. Ocho de las cuentas analizadas, no alcanzan ni un tweet por año. 
Dos de las cuentas muestran, por mucho, mayor actividad y frecuencia de publicación, habiendo tweeteado ambas en el último mes. Se trata de las cuentas@Danny_Arevalo17 y@SilviaGaibor05 con 237 y 616 Tweets por año, respectivamente, ambos docentes, el primero de la UEM 2 de Agosto, y la segunda de la UEM Carmelina Granja Villanueva.

En una búsqueda en Twitter, los tweets destacados de la cuenta (a) SilviaGaibor05 son retweets de cuentas de personas y colectivos alineados a movimientos políticos ecuatorianos, varias de ellas vinculadas a la educación, incluyendo referencias a la inauguración de nuevas UEM y el colectivo Red de Maestros por la Revolución ciudadana.En la cuenta@Danny_Arevalo17, dos de los tweets destacados se refieren directamente a la UEM 2 de Agosto, compartiendo dos imágenes, una del cuerpo docente y otra de una tarde de integración en la institución.

En resumen, se infiere que las cuentas institucionales de las UEM, y aquellas que integran las mismas a su identidad son pocas y en su gran mayoría muestran baja visibilidad y actividad y no poseen muchos seguidores en las redes, evidenciándose que este canal tiene un rol secundario entre las estrategias de comunicación que permiten construir la presencialidad institucional, entendida esta como entramado complejo de relaciones y, en particular, la relación institución - comunidad.

En cuanto a la segunda dimensión analítica, las UEM son objetos de atención periodística al ser instituciones de creación reciente, todas son noticia en su inauguración, al menos a nivel local. Periódicos tradicionales, portales de información web, entidades gubernamentales y autoridades públicas muestran estos eventos a través de sus cuentas de Twitter, los mensajes suelen estar acompañados de enlaces a videos, o ampliaciones noticiosas en páginas institucionales, en algunos casos las autoridades de las nuevas instituciones participan también en la cobertura. Las inauguraciones de las primeras UEM, por ejemplo, tuvieron presencia del presidente de la República de ese entonces, así como representantes del Ministerio de Educación - Mineduc.

La información obtenida conformó una red de 129 nodos, incluyendo 42 cuentas de usuario que tweeteaban o retweeteaban mensajes vinculados a las UEM, 43 tweets, incluyéndose re-tweeteos, 13 hashtags y 31 vínculos a imágenes, esos nodos se conectan entre sí a través de 179 aristas. En el caso de esta red, la densidad reportada es de 0,011; es decir que las 179 conexiones reportadas son apenas el 1,1\% de las potenciales conexiones de la red analizada, en resumen, la red presenta baja densidad y poco relacionamiento entre los actores existentes. 
Adicionalmente, observamos pocas vinculaciones directas entre nodos, siendo una red compacta y de baja densidad, esto significa que los diálogos alrededor de las unidades, no solo que involucran a pocas personas e información, sino que los vínculos o diálogos son a la vez pocos y débiles, de tipo coyuntural. (RECUERO; BASTOS; ZAGO, 2015)

La modularidad en la red analizada corresponde a 0,817 ; detectándose 10 sub-comunidades en la red, dos de las cuales están muy vinculadas. Las siguientes figuras, muestran los arreglos gráficos de cada una de esas sub-comunidades.

ILUSTRACIÓN 2. Sub-comunidad @goberloja

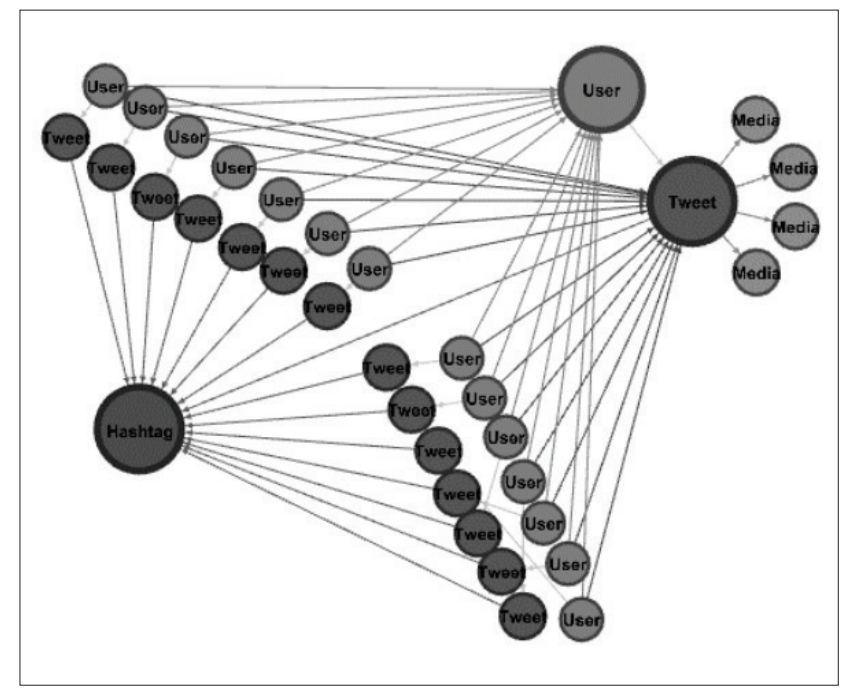

Elaborado por los autores.

El tipo de nodo viene en la figura y el tamaño de los mismos corresponde al grado de Soporte, es decir, los nodos mayores serán aquellos con más vinculaciones recibidas. En la ilustración 2, se observa que la sub-comunidad gira alrededor de un usuario y sus acciones principalmente.

El mayor nodo de usuario es la cuenta@goberloja,, siendo su tweet y su hashtag \#loja, también los más soportados. El tweet es retweeteado por el resto de miembros de la sub-comunidad, formada por 14 cuentas personales. Este grupo tiene un carácter multiplicador e informativo, ya que lo expedido por la Gobernación de la Provincia de Loja es difundido por los demás usuarios, en ningún caso se adiciona un comentario al re-tweet. El tweet en cuestión, se refiere a 
la visita de representantes de la Gobernación, a la graduación del bachillerato intensivo, ofrecido por la UEM Bernardo Valdivieso, cuya cuenta, no es citada en el tweet. Las cuatro medias del tweet son imágenes de la mesa directiva y otras capturas con los graduados.

ILUSTRACIÓN 3. Sub-Comunidad @goberchimborazo

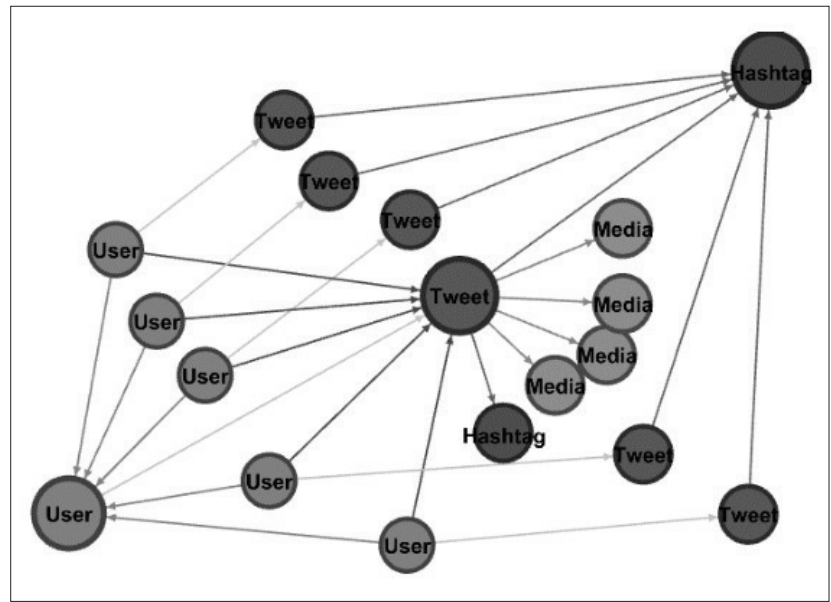

Elaborado por los autores.

El caso de esta sub-comunidad es idéntico a la primera, en ese caso,la cuenta@goberchimborazo,de la Gobernación de la Provincia de Chimborazo, emitió un tweet informando una reunión de la Gobernadora con la Junta Parroquial de la población de Huigra y representantes de la UEM. El Tweet está acompañado de dos hashtags, \#Chimborazo y \#Huigra y no especifica la institución considerada. Se intuye que la reunión pudo ser con representantes de las tres UEM existentes en la provincia de Chimborazo: UEM Penipe, 27 de Febrero y Guano, o una de ellas. Los cuatro elementos multimediales del Tweet original corresponden a fotos de las reuniones citadas en el Tweet, realizadas en la misma gobernación. 
ILUSTRACIÓN 4. Sub-comunidad "Zonal"

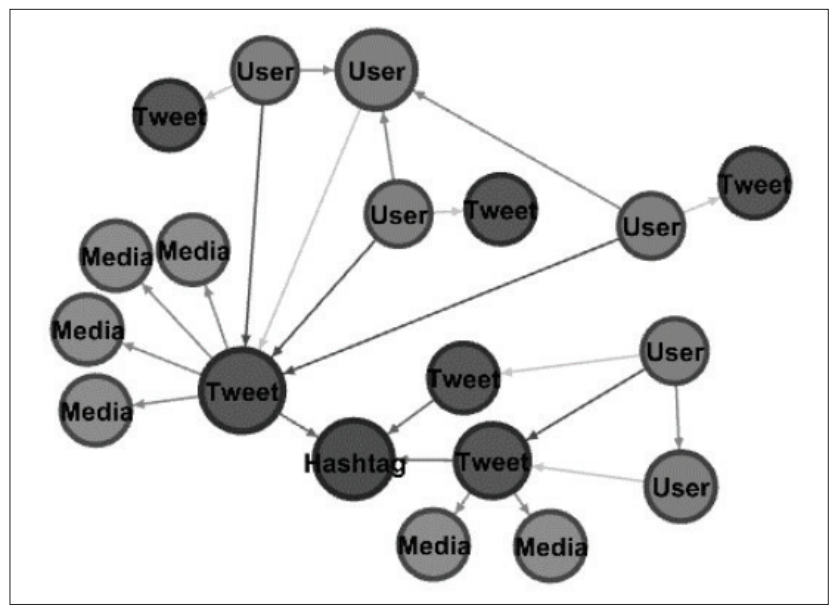

Elaborado por los autores.

La tercera comunidad tiene dos tweets originales, re-tweeteados tres y una vez respectivamente, razón por la cual el primer tweet tiene mayor tamaño al tener mayor grado de entrada. Todos los usuarios de esta sub-comunidad tienen similar influencia y soporte, la comunidad debe su nombre, a que tres de las seis cuentas que conforman el cluster son las cuentas institucionales@justicia_zona7,@justicia_zonal6y @educacionz7_ec. Las mismas corresponden a zonas territoriales del Ecuador creadas para la planificación de políticas públicas mejor direccionadas localmente. Cada zona agrupa, principalmente, provincias del país, y tienen representaciones por cada Ministerio, por ejemplo, zonas de educación o justicia.

El primer tweet original, emitido por@justicia_zona7y retweeteado por dos cuentas personales y la cuenta@educacionz7_ ec, se refiere a la obra teatral "Cristales" del grupo "Sunset" de la UEM Bernardo Valdivieso, institución que aparece por segunda vez en los mensajes capturados, pero, nuevamente, su cuenta no es mencionada; el tweet se acompaña de cuatro imágenes, dos de la obra de teatro y otras dos del público asistente.

E1 tweet de@justicia_zona7 contiene un hashtag, \#NoviazgoSinViolencia, que sirve de vínculo con la otra parte de esta sub-comunidad, al compartir este hashtag con un tweet emitido por la cuenta@justicia_zonal6y retweetteado una vez. El tweet trata de la obra de teatro "Amor con respeto" de la UEM Paiwara, vinculada directamente al hashtag "NoviazgoSinViolencia". Este tweet se acompaña de dos imágenes, mostrando capturas de la obra en cuestión. 
ILUSTRACIÓN 5. Sub-comunidad @ciudadyachay

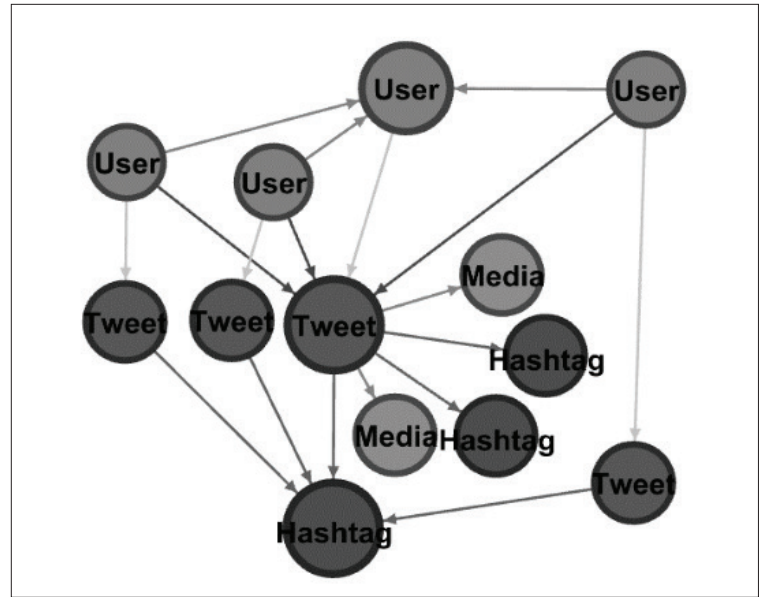

Elaborado por los autores.

Esta comunidad toma su nombre del usuario@ciudadyachay que emitió un Tweet retweeteado por tres cuentas personales. El mismo informa del Segundo Festival de la Canción en Inglés, realizado en la UEM Yachay. Este tweet se acompañó de dos imágenes del festival y tres hashtags: \#Felizjueves, \#empresaspublicas y \#ucuqui, este último mal escrito, pues se refiere a San Miguel de Urcuquí, población donde se ubica la UEM citada.

ILUSTRACIÓN 6. Sub-comunidad @Salud CZ2

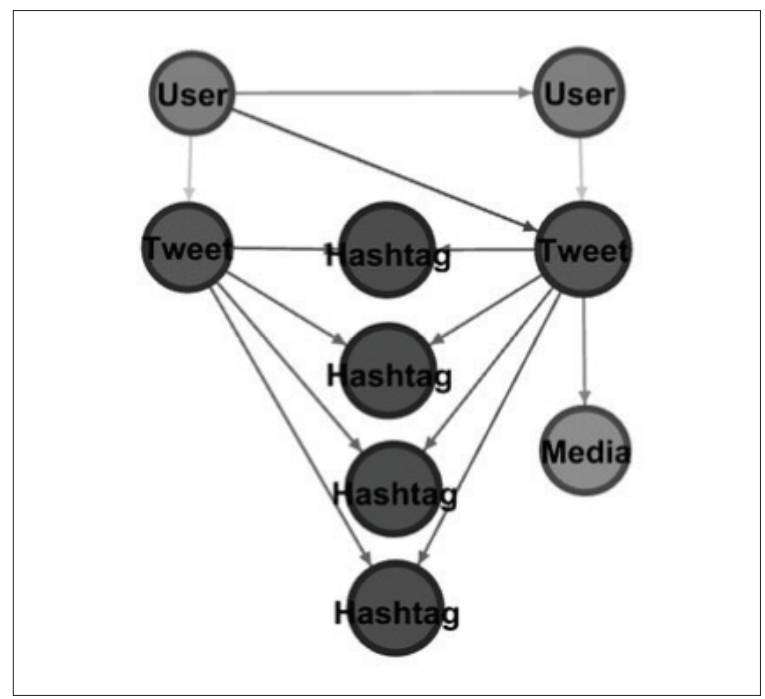

Elaborado por los autores. 
Esta sub-comunidad se enfoca en la cuenta@Salud_CZ2, correspondiente a la Coordinación Zonal 2 del Ministerio de Salud, que agrupa las provincias de Napo, Orellana y la ruralidad de Pichincha. Esta sub-comunidad es la que más hashtags posee, incluyendo: \#archidona, \#tena, \#dd15d01 y \#carlosjulioarosemenatola, los dos primeros se refieren a ciudades, el tercero se refiere a la identificación de la Dirección Distrital de Salud de Tena y el cuarto hashtag es el nombre del Comité de Salud local.

El tweet refiere el apoyo, a través de oficio escrito, por parte del Rector de la UEM Chontapunta a la gestión de la Ministra de Salud. La imagen que acompaña al tweet es una foto del oficio enviado a través de medios oficiales.

ILUSTRACIÓN 7. @politicaec

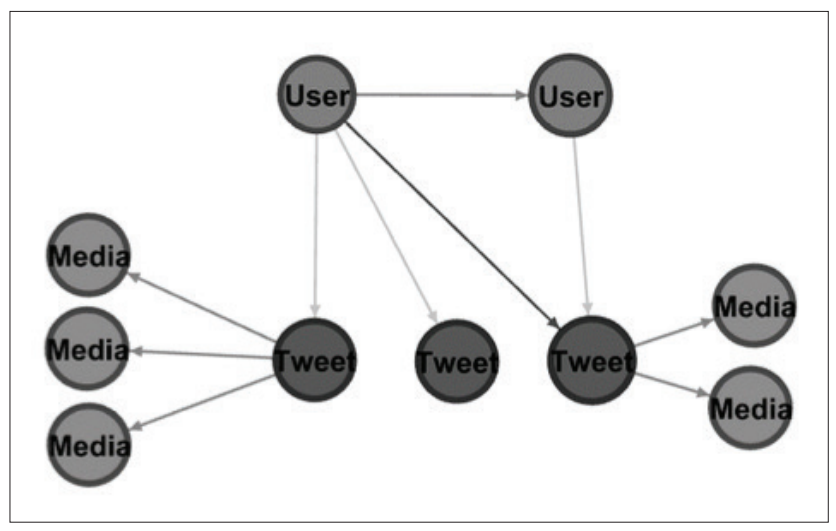

Elaborado por los autores.

La sub-comunidad@politicaec es un caso similar a lo visto anteriormente, es decir la emisión de un tweet y su posterior retweeteo por otras cuentas. Lo particular de esta sub-comunidad es que además del retweet se hace un nuevo tweet con el mismo texto con una imagen adicional del original. El tweet origen narra un recorrido realizado en la UEM Eloy Alfaro por personeros del Ministerio de Educación (@Educacion_Ec) y la Secretaria Nacional de Gestión de la política (@politicaec),para garantizar que la infraestructura esté en óptimas condiciones para los estudiantes. El texto se acompaña de dos imágenes de personeros reunidos durante la revisión. 
ILUSTRACIÓN 8, 9 Y 10. Subcomunidades @municipiojama, @deporte_cz2 y @educacionz2 ec

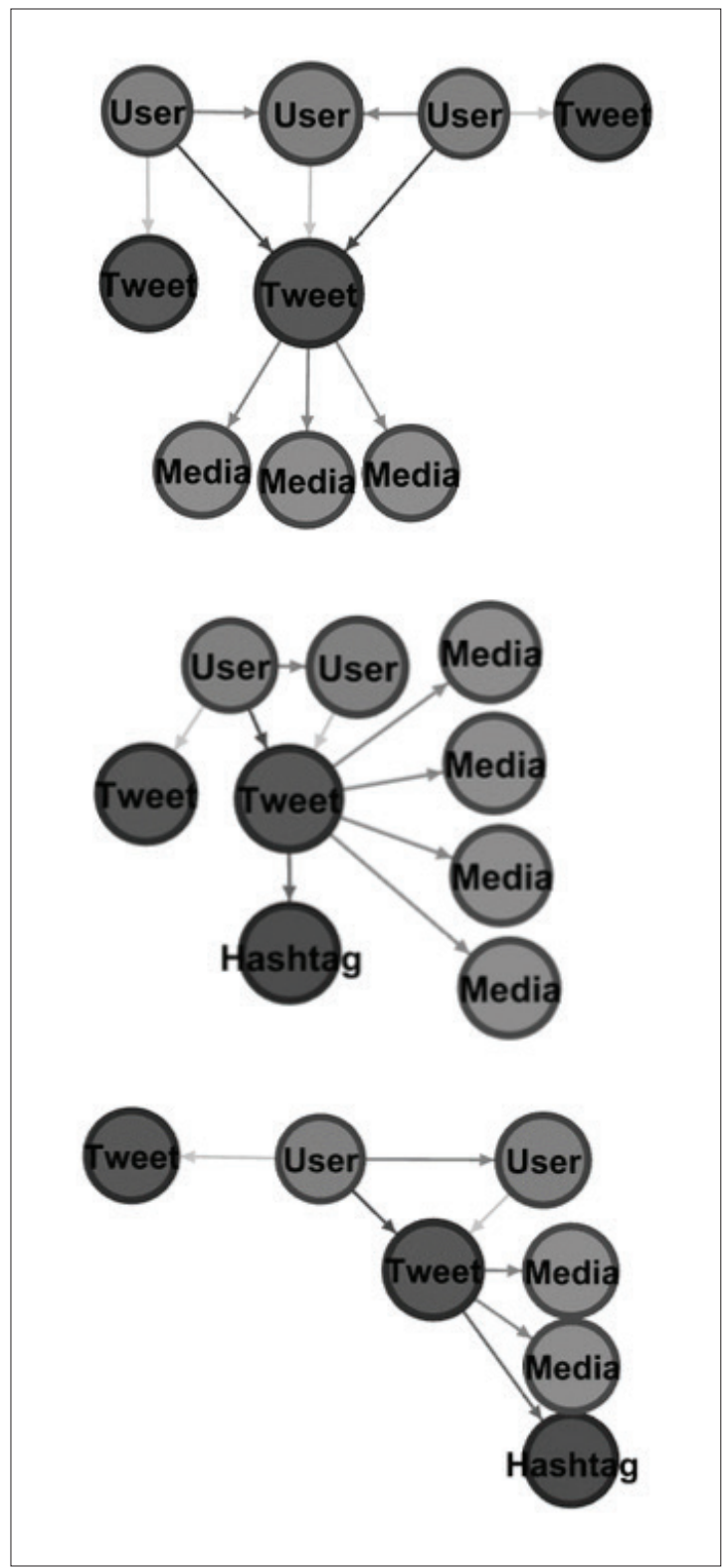

Elaborado por los autores.

Las últimas sub-comunidades encontradas demuestran el mismo comportamiento ya visto, pero en su mínima expresión, es 
decir la emisión de un Tweet sobre las UEM y el retweet del mismo por uno o dos usuarios. El nombre asignado a cada sub-comunidad corresponde a las cuentas que emitieron el tweet original. El tweet de la primera comunidad narra la presencia del Alcalde de Jama en la UEM homónima, en la ceremonia de graduación de bachillerato. Tres imágenes del evento acompañan al texto.

En cuanto a la segunda comunidad, el tweet se refiere a la presencia de la Coordinación Zonal 2 del Ministerio de Deporte en la inauguración de los II Juegos deportivos de la UEM Jorge Rodríguez Román. Las imágenes muestran a estudiantes en atuendos deportivos y se usa el hashtag \#ElDeporteNosune.

Sobre la tercera sub-comunidad, el tweet informa que 33 estudiantes universitarios realizarán sus práctica pre-profesionales en la UEM Nuevo Rocafuerte. Dos imágenes muestran a los estudiantes en las instalaciones de la institución, y se coloca el hashtag \#PorlaEducacionEc.

\section{CONCLUSIONES}

Si consideramos que las UEM como iniciativa educativa tienen entre sus pilares o ejes de acción la participación y el uso de tecnologías, a marzo del 2018, la presencialidad de las UEM en la plataforma Twitter tiene varios espacios de fortalecimiento, en especial si se visualiza esa plataforma como medio de vinculación y diálogo con actores locales y externos, así como de intercambio y generación de conocimiento.

De las 97 UEM en funcionamiento al momento de este estudio, apenas once instituciones tienen cuenta en Twitter para visibilizar a la comunidad los logros institucionales y relacionarse con su entorno de influencia. Por otra parte, la actividad y visibilidad de las cuentas son bajas, excepto en dos casos: la cuenta de la UEM Carlos Alberto Aguirre Avilés con 563 Tweets por año y la cuenta de la UEM Réplica Juan Pío Montúfar con 508 Tweets por año.

Los resultados de este estudio se corresponden con un análisis sobre la presencialidad de las UEM en Facebook, donde se determina baja participación oficial o institucional en esa plataforma, con flujo de información bajo, donde las publicaciones se dan de forma semestral (CEVALLOS; FICOSECO; COUTO, 2018, p.57). Así, tanto en la plataforma de Facebook como en Twitter se observa que la mayoría de cuentas con referencia a las UEM no son oficiales, evidenciándose que la presencialidad institucional actualmente está siendo construida por iniciativas personales de la comunidad educativa. 
Estos hallazgos evidencian que existe un sector de la comunidad educativa que detenta una apropiación significativa de la plataforma, dado que le atribuye sentido y construye participación em la misma (CASTELLS, 2015), y que la usa en relación a la UEM, en el caso de Twitter para describir y crear identidad, o generar visibilidad. Esa apropiación comunitaria es una base requerida para emprender procesos de relación con la comunidad, potencialmente aprovechable para fomentar la comunicación multidireccional y el involucramiento amplio em temas educativos com base en ambientes participativos posibilitados por las tecnologías digitales.

En cuanto al carácter de la información generada en las plataformas Facebook y Twitter, se observó que mientras en la primera existía diversidad de tópicos, desde la búsqueda de empleo hasta la organización de eventos recreativos, por otro lado en Twitter la presencialidad de las UEM sigue una temática más homogénea.

Por otra parte, las UEM podrían ser objeto de dinamización y diálogo de la reflexión educativa en el país, en especial cuando esta iniciativa tiene carácter nacional y ha alcanzado prácticamente toda la geografía del Ecuador, en los diez años transcurridos desde la primera UEM inaugurada. Sin embargo, durante el periodo analizado, las menciones a las UEM se refieren a eventos y acciones de aprendizaje, que si bien importantes no dejan de ser coyunturales.

En los datos obtenidos se puede establecer una gran atomización del abordaje de las UEM, las diversas conversaciones no salen de las localidades de cada comunidad, no existen vínculos o relaciones directas al hablar de las mismas, a tal punto que el hashtag \#UEM, que es usado por instituciones educativas de Brasil y España, cuando es referido al territorio ecuatoriano, no aparece en la búsqueda de destacados de Twitter, desde el 2015.

La atomización se verifica también, cuando los tweets que hacen referencia a las UEM y sus actividades no utilizan las cuentas de las mismas, como se observó dos veces en lo referente a la UEM Bernardo Valdivieso. Mantener un procedimiento de cruces y referencialidad entre las coordinaciones zonales de educación y las UEM podría generar una red más densa, que haga llegar las experiencias entre estas instituciones y la comunidad.

Finalmente, al analizar las sub-comunidades de la red generada, la mayoría de menciones a las UEM son tópicos coyunturales, sometidos al campo político, particularmente en informes de labores a la sociedad por parte de diferentes entidades del Estado ecuatoriano, como coordinaciones zonales o Ministerios. 
Este estudio generó informaciones para profundizar el conocimiento acerca de la presencialidad de las UEM en Twitter, que permiten comprender cómo esta plataforma tiene posibilidades de ser medio de diálogo y reflexión educativa y se inserta en un más amplio abanico de plataformas digitales y de espacios institucionales de presencia tradicional, que conforman en conjunto la visibilidad pública de las UEM y a su vez determinan sus posibilidades de interacción con las comunidades

Consideramos que una presencialidad empoderada de las UEM en esta y otras plataformas posibilitadas por las TIC puede elevar la generación de conocimiento, a través de la vinculación con la comunidad y el intercambio de experiencias con instituciones similares, así como de otro tipo y latitudes. Al mismo tiempo, permite la generación de espacios relativamente novedosos para la interacción entre comunidades y escuela, lo que potencialmente podría introducir elementos innovadores en el rol social de esas instituciones educativas tradicionales, desde su capacidad para atender demandas puntuales de la comunidad hasta la eficiencia en la planificación y ejecución de acciones que las atiendan.

\section{BIBLIOGRAFÍA}

CASTELLS, M. Redes de indignación y esperanza. 1a. ed. Barcelona: Alianza, v. I, 2015, p. $1-13$.

CEVAllos, G.; FICOSECO, V. \& SOUZA COUTO, E. La presencia de las unidades educativas del mileno (UEM-Ecuador) en Facebook. Praxis Educativa, XXI(1), 2018, p. 15-32.

COHEN, D.; ASÍN, E. Sistemas de información para los negocios. Un enfoque de toma de decisiones. Tercera. ed. Buenos Aires: McGraw-Hill, v. I, 2000.

HANNEMAN, R. Propiedades básicas de las redes y de los actores. In: RIVERSIDE, U. D. C. Introducción a los métodos del análisis de redes sociales. Primera. ed. California: Universidad de California Riverside, v. I, 2014. Cap. 5, p. 50-76.

HINE, C. Ethnography for the internet. Embedded, embodied and every day. 1a. ed. Norfolk: Bloomsbury, v. I, 2015.

KIMMONS, R., VELETSIANOS, G., \& WOODWARD, S. Institutional uses of twitter in u.s. higher education. Innovative Higher Education, 42(2), 2017, p. 97-113.

LARROSA, J. Análisis de Redes Sociales, 2012. Disponivel em: < http://ars-uns.blogspot. com/2016/09/densidad-de-red-que-es-y-como-se-calcula.html>. Acceso en: 16 Marzo 2018.

LEMOS, A. A comunicação das coisas, teoria ator-rede e cibercultura. Primera. ed. San Pablo: Annablume, v. I, 2013. 
LÉVY, P. The Semantic Sphere: Computation, Cognition and Information Economy. 1a. ed. London: ISTED Ltd., v. I, 2014.

MINAYO, Maria Cecília de Souza (Org). Pesquisa social: teoría, método e criatividade. $32^{a}$ edição. Petrópolis, Vozes, 2011.

MINISTERIO DE EDUCACIÓN DEL ECUADOR. Página oficial del Mineduc. UEM en funcionamiento, 2010. Disponivel em: <http://educacion.gob.ec/uem-enfuncionamiento/>. Acceso en: 2016 Mayo 14.

MINISTERIO DE TELECOMUNICACIONES Y SOCIEDAD DE LA INFORMACIÓN DEL ECUADOR. Página Oficial del Mintel. 91\% de ecuatorianos utiliza las redes sociales en su teléfono inteligente, 2017. Disponivel em: <https://www.telecomunicaciones.gob. ec/91-de-ecuatorianos-utiliza-las-redes-sociales-en-su-telefono-inteligente/> Acceso en: 2018 Noviembre 16.

NEGROPONTE, N. Ser digital. Segunda. ed. Buenos Aires: Atlántda, v. I, 1995.

PÉREZ BELTRÁN, J. E.; VALERIO UREÑA, G.; RODRÍGUEZ-ACEVES, L. Análisis de redes sociales para el estudio de la producción intelectual en grupos de investigación. Perfiles educativos, México D.F., v. 37, n. 150, 2015, p. 1-15.

RECUERO, R.; MARCO, B.; GABRIELA, Z. Análise de Redes para Mídia Social. Primera. ed. Porto Alegre: Sulina, v. I, 2015.

UNESCO. Uso de TIC en Educación en América Latina y el Caribe. Montereal. 2013.

\section{NOTAS}

${ }^{1}$ Estudios financiados por la Coordenação de Aperfeiçoamento de Pessoal de Nível Superior - Brasil (CAPES) - Código de Financiamiento 001.

2 Audiense es un programa privativo que permite reunir cuentas de Twitter, según las configuraciones del usuario, permitiendo generar estadísticas grupales sobre entradas, tweets, entre otros. Disponible en: https://es.audiense.com/

${ }^{3}$ Gephi es un programa que permite la modelación de mapas de red, su uso específico en este estudio se describe en la metodología. Disponible en: https://gephi.org/

${ }^{4}$ Twitter verifica si el usuario es quien dice ser, este proceso, si bien puede solicitarse, se aplica específicamente a sujetos que aglutinan gran cantidad de seguidores, y por tanto poseen gran visibilidad y posibilidades de crear tendencias, los llamados influencers. Las cuentas verificadas por Twitter se identifican por un ícono de listón azul que coloca la plataforma en el perfil de la cuenta y sus publicaciones.

${ }^{5}$ Twlets permite descargar la información de cuentas, tweets y grupos en formato de hoja de datos, a través de una cuenta de Twitter. Disponible en: http://twlets.com/

${ }^{6}$ Gephi no captura respuestas a los tweets.

${ }^{7}$ Disponible en: http://www.bernardovaldivieso.edu.ec/ 
Submetido: 24/04/2018

Aprovado: 19/12/2018

Contato:

Gabriel Francisco Cevallos Martínez Martín Ochoa N59-60 y Pasaje "E"

Quito | Pichincha | Ecuador Código postal: EC170528 